\title{
Supporting Information: Structure and Dynamics of an Ionic Liquid Mixture Film Confined by Mica
}

\author{
Waruni V. Karunaratne and Claudio J. Margulis* \\ Department of Chemistry, University of Iowa, Iowa City, Iowa 52242, United States \\ E-mail: claudio-margulis@uiowa.edu
}




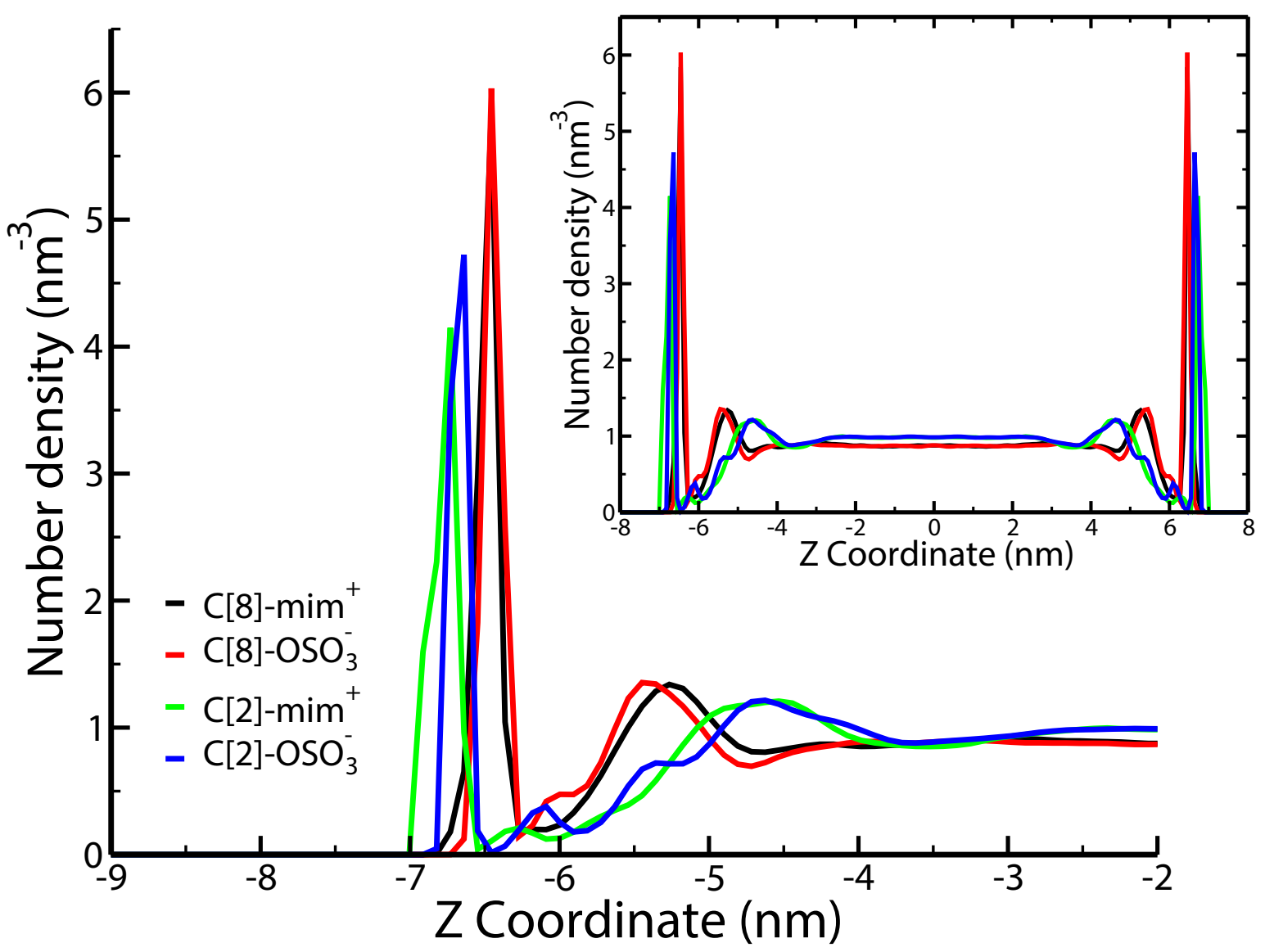

Figure S.1: Symmetrized density profile for the centers of mass of IL components in our mica-confined film. The full symmetrized density profile is shown in the inset. These distributions are used to define the slab locations for the calculation of survival probability time correlation functions as well as lateral mean square displacements. 


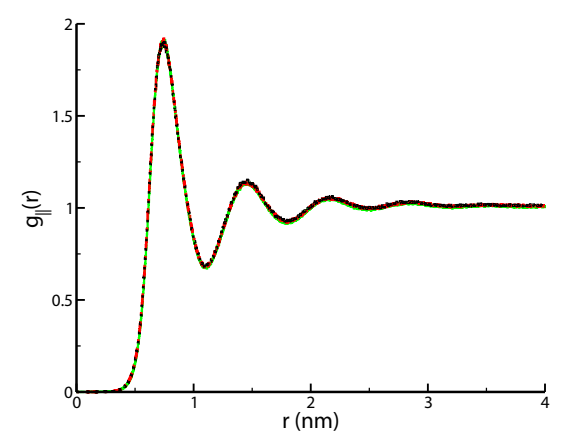

(a)

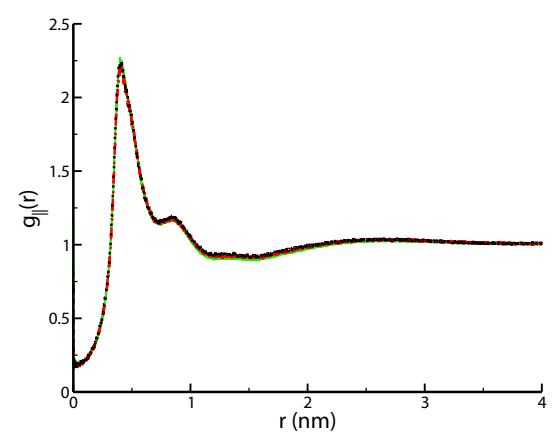

(b)

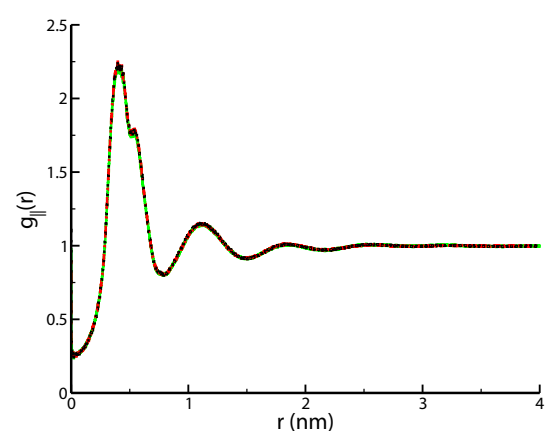

(c)

Figure S.2: Comparison of TRDFs in the unperturbed bulk phase (black) against bulk-like regions of a vacuum-surrounded film $-{ }^{1}$ (red) and mica-surrounded film- (green) for (a) the $\mathrm{S}-\mathrm{S}$ correlation of all anions (b) the terminal $\mathrm{C}$ correlation of all long tail ions and (c) the $\mathrm{N}-\mathrm{O}$ correlation between $\mathrm{C}[2]-\mathrm{mim}^{+}$and $\mathrm{C}[2]-\mathrm{OSO}_{3}^{-}$ions. Subfigure (a) links anions of all sizes, whereas (b) only links the larger ions and (c) only the smaller ions. In all cases, the distributions are nearly identical implying that convergence to structural bulk behavior has been achieved at the center of our films.

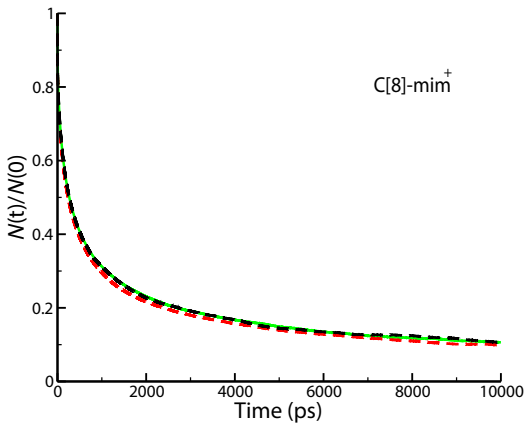

(a)

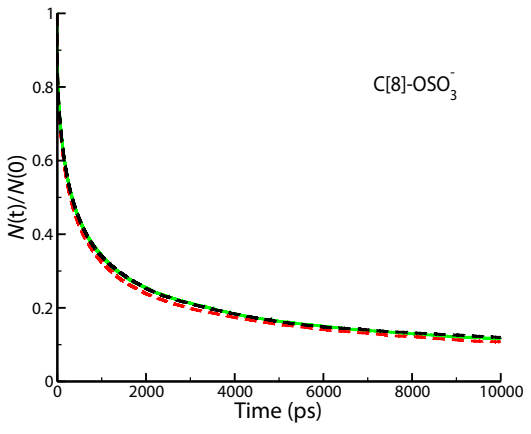

(b)

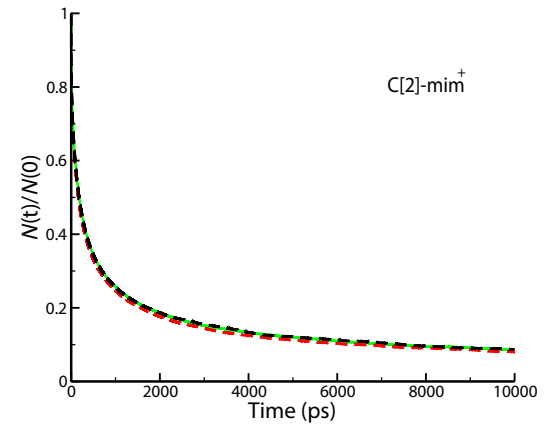

(c)

Figure S.3: Comparison of survival probability time correlation functions for different ions in slabs of actual bulk in comparison to the bulk-like regions of vacuum- and mica-confined films color-coded in black, red and green respectively. All functions are nearly identical implying that for this property bulk behavior has been achieved. 


\section{References}

(1) Wu, F.; Karunaratne, W. V.; Margulis, C. J. Ionic Liquid Mixture at the Vacuum Interface and the Peaks and Antipeaks Analysis of X-ray Reflectivity. J. Phys. Chem. C 2019, 123, 4914-4925. 